\title{
Facilitating Human-Mobile Robot Communication via Haptic Feedback and Gesture Teleoperation
}

\author{
YUHANG CHE, Stanford University, CA \\ HEATHER CULBERTSON, University of Southern California, CA \\ CHIH-WEI TANG and SUDIPTO AICH, Ford Motor Company \\ ALLISON M. OKAMURA, Stanford University, CA
}

\begin{abstract}
In this article, we present a bi-directional communication scheme that facilitates interaction between a person and a mobile robot that follows the person. A person-following robot can assist people in many applications including load carrying, elder care, and emotional support. However, commercially available personal robot systems usually have limited sensing and actuation capabilities. They are not expected to function perfectly in complex environments, and human intervention is required when the robot fails. We propose to use a holdable mechatronic device to reduce the user's effort in communication and enable natural interaction during the intervention. Our design of the holdable device consists of two parts: a haptic interface that displays touch cues to convey the robot's failure status via asymmetric vibrations, and a command interface for teleoperating the robot follower with hand gestures. We experimentally evaluated the device and the communication strategy in two sets of user studies with a controlled environment and a physical robot follower. Results show that with the proposed method, users are able to perform their tasks better, respond to robot failure events faster, and adjust walking speed according to the robot's limitations. We also demonstrate that users can successfully teleoperate the robot to avoid obstacles when navigating in challenging environments.
\end{abstract}

CCS Concepts: • Human-centered computing $\rightarrow$ Interaction paradigms; Haptic devices; User studies; - Computer systems organization $\rightarrow$ Robotics;

Additional Key Words and Phrases: Human robot interaction, mobile robots, human-following robot

\section{ACM Reference format:}

Yuhang Che, Heather Culbertson, Chih-Wei Tang, Sudipto Aich, and Allison M. Okamura. 2018. Facilitating Human-Mobile Robot Communication via Haptic Feedback and Gesture Teleoperation. ACM Trans. Hum.Robot Interact. 7, 3, Article 20 (November 2018), 23 pages.

https://doi.org/10.1145/3243503

\section{INTRODUCTION}

Mobile robots have the potential to assist humans in many domains, including medicine, the military, recreation, and industry [43]. In this article, we focus on the application of personal assistive

This work was supported in part by the Ford Motor Company and Stanford University MediaX.

Authors' addresses: Y. Che and A. M. Okamura, Stanford University, Mechanical Engineering, Stanford, CA, 94305; emails: \{yuhangc, aokamura\}@stanford.edu; H. Culbertson, University of Southern California, Computer Science, Los Angeles, CA, 90007; email: hculbert@usc.edu; C.-W. Tang and S. Aich, Ford Motor Company, Palo Alto, CA, 94304; emails: \{ctang32, saich\}@ford.com.

Permission to make digital or hard copies of all or part of this work for personal or classroom use is granted without fee provided that copies are not made or distributed for profit or commercial advantage and that copies bear this notice and the full citation on the first page. Copyrights for components of this work owned by others than the author(s) must be honored. Abstracting with credit is permitted. To copy otherwise, or republish, to post on servers or to redistribute to lists, requires prior specific permission and/or a fee. Request permissions from permissions@acm.org.

2018 Copyright is held by the owner/author(s). Publication rights licensed to ACM.

ACM 2573-9522/2018/11-ART20

https://doi.org/10.1145/3243503 
(a)

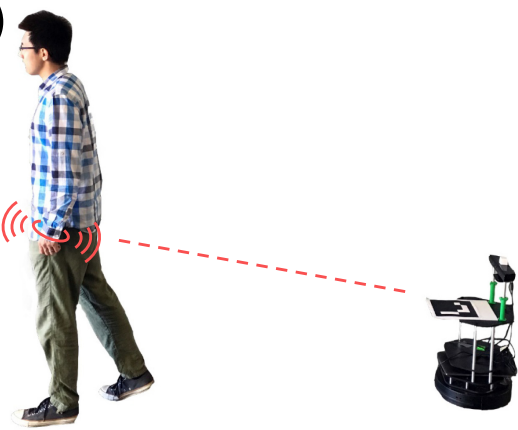

(b) Communication Device

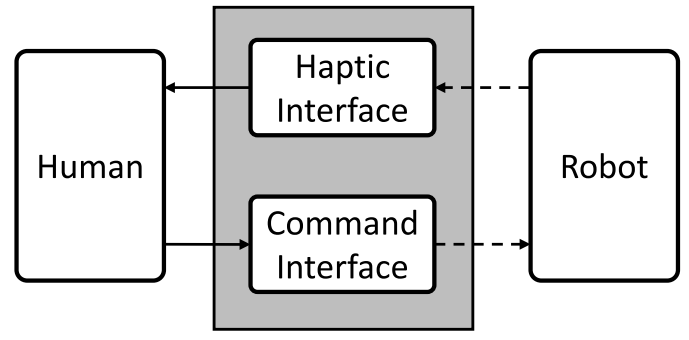

Fig. 1. (a) A human walking with a robot follower. The user and the robot can communicate with each other via a holdable mechatronic device. (b) System block diagram demonstrating the relationship of different components of the human-robot communication system. The shaded blocks are parts of the communication device. Arrows indicate the direction of information flow. Solid arrows represent physical connections and dashed arrows represent non-physical connections.

robots, in particular, robots that can follow people and help with tasks like carrying loads. To follow and assist people is one of the most common tasks for intelligent service robots. However, this seemingly simple task, which requires the ability to track moving people, understand the environment, and plan appropriate actions [45], is not always straightforward. Each of these areas is being actively researched, and to the best of our knowledge, there is no complete solution for any of these problems. As a result, commercially available products such as robotic golf caddies usually implement simple person-following methods and are incapable of active obstacle avoidance or adapting to changes in the environment.

Many researchers are working on sensors and algorithms that improve the reliability of mobile service robots and decrease costs. In this work, we take a different route: instead of making the robot smarter, we propose to bring the human user into the loop when the robot fails to follow the user. This is accomplished with a holdable mechatronic device that enables bi-directional communication between the user and the robot. The concept and the system components are presented in Figure 1. When the robot fails to follow the user because of obstacles or other unexpected conditions, the user will be notified by the haptic interface of the communication device. The user can then use the command interface to teleoperate the robot to a safe location and to direct it to resume following. The main advantages of the proposed approach are:

- Our method is not an alternative to existing human-following methods. It can be used in combination with almost all robot platforms and following algorithms.

-It augments the connection between the user and the robot follower and enables the robot to recover from various failures with a human in the loop.

Non-contact human-robot interaction can be achieved via different communication channels including visual [5], audio [40], and haptic feedback [34, 36, 39]. In the case of interacting with human-following robots, haptic feedback has certain advantages over visual and audio feedback. In a typical robot follower setup, the robot will be behind the user most of the time. Therefore, any visual cues displayed by the robot will be hard for the user to observe. Wearable devices can be made to display visual cues, but will require too much user attention. In fact, the user's visual channel is usually overloaded with information from the environment. Similarly, the user's audio channel may also be overloaded if the environment is noisy or if the user needs to talk. The haptic channel, on the other hand, is often underutilized when the user is walking. In addition, haptic 
feedback is private, noticeable, and ideally does not require mental models to parse the information. Our holdable haptic interface uses asymmetric vibrations to render different directional cues, which alert the user of failure events and the relative direction of the robot.

To enable the user to take control of the robot when if fails, we implemented an intuitive teleoperation interface with on-body inertial measurement units (IMU). The user can control the robot by tilting his or her hand. The design principle of the command interface is to allow for natural interaction while providing the user with necessary control of the robot.

The main contributions of this article are:

(1) A novel bi-directional communication scheme that brings the human into the loop in mobile robot following applications. A holdable mechatronic device was designed to realize the communication.

(2) Experimental evaluation of the proposed approach with user studies, which demonstrated that the communication device can effectively alert the user of robot failure events, help the user to adapt to the robot's limitations, and enable the robot to function in challenging environments.

The rest of the article is organized as follows. Section 2 reviews methods for human-following, human-mobile robot interaction, and wearable haptic technologies. Section 3 describes the robot follower algorithm and behavior. Section 4 presents the design of the communication device. In Sections 5 and 6, we evaluate the proposed communication approach and device with two sets of user studies. The results are discussed in Section 7. Finally, in Section 8, we provide a summary of the work.

\section{BACKGROUND}

\subsection{Person-Following Robot}

Person-following is a common task for service and assistive robots. Researchers have developed person-following systems for different applications, including helping elderly people who suffer from cognitive impairment [15,32] and carrying oxygen therapy tanks for patients [41]. According to Ref. [45], a robot follower should perform the following tasks: find the specified person, follow the person, avoid obstacles, and resume tracking after occlusion. We will briefly review existing work in these areas.

For human detection and tracking, one typical method is to use pattern detection, choosing clothing texture and human body parts as template patterns to be detected and identified [18, 45]. Researchers have also combined information from different sensing modalities: Ref. [19] developed methods to integrate two types of range finders to identify and track a human; Ref. [21] combined an omnidirectional camera and a laser to obtain robust tracking in an outdoor environment. In recent years, combined RGB image and depth sensing has enabled skeleton tracking, which can be used in combination with other visual detectors and filters for human tracking [4]. In the case of occlusion, an Extended Kalman Filter (EKF) based method has been used Ref. [33] to infer the human leader's position with a motion model.

Researchers have also studied methods to control robots to follow humans. A spring model was introduced in Ref. [26], where the robot follower was connected to the human by a virtual spring that can linearly deform and bend. In Ref. [14], the authors presented two methods of humanfollowing: direct-following and path-following. In the direct-following mode, the robot always drove directly toward the human, while in the path-following mode, the robot followed the exact path that the human took. The authors of Ref. [46] developed a force field method to drive the robot follower toward the human and away from obstacles. Closely related to human-following is the 
topic of formation control of robot teams [11, 12, 22]. One of the popular methods for formation control is leader-follower, where one robot serves as the leader and follows a predefined path, while the other robots maintain desired distances and orientations to the leader $[6,11]$. These algorithms can be adjusted and applied to human-following robots.

The task of human-following can also be treated more generally as trying to make the robot reach a dynamic target location. In this context, a wide variety of motion planning algorithms can be used to move the robot to the desired locations and avoid obstacles. While there are many possible ways to improve the intelligence and performance of the person-following robot itself, this is not an objective of our work. Our goal is to augment the human-robot follower system with bi-directional communication, so that an imperfect robot follower can function robustly with a human in the loop.

\subsection{Human-Mobile Robot Interaction}

Several groups have studied human-robot interaction in human-following robot systems. The authors of Refs. [35] and [36] designed a vibrotactile haptic bracelet for interaction in human-robot teams. They extended the algorithm in Ref. [6] to guide the human along trajectories that are feasible for leader-follower formation tasks. The authors also applied the same haptic bracelet to a robot leader-human follower application and showed that the robot can successfully guide the users to target locations [34]. A similar interaction scheme is proposed in Ref. [39], where the human leader teleoperated a team of robots that collaboratively manipulate an object. The teleoperation was realized using a leader-follower formation control algorithm, and haptic feedback was used to inform the user of the states of the robots (in transition or stable). Our work also aims to use haptic feedback to facilitate human-mobile robot interaction, but is different in a number of aspects. The aforementioned works focus on the use of vibrotactile feedback to improve the accuracy of formation control, and the information provided by haptic feedback is tightly coupled with their formation algorithms. In contrast, our approach uses haptic feedback to communicate general failure events and to indicate the relative position of a robot. We decouple the feedback from the human-following algorithm. In addition, we propose a bi-directional communication strategy that allows information to flow from the user to the robot and vice versa, while the aforementioned works focus only on haptic feedback to the user.

Another relevant topic is "symbiotic" human-robot interaction, where the robots can ask for help from humans to overcome their limitations [29-31]. In those works, the authors proposed a planning framework that allows the robot to request help to complete actions that it could not complete otherwise. The application that we focus on (person-following robot) is quite different, but the idea of "symbiotic" relationship motivates us to develop the proposed communication strategy: the robot follower serves the user, and the user can help the robot when necessary.

Finally, Refs. [23] and [24] present a semi-autonomous trajectory generation algorithm that allows a human to interactively modify the trajectory. Haptic feedback provided information of the mismatch between the user specified path and the autonomously-corrected path. This is a good example of using a human-in-the-loop approach to improve autonomous performance. However, the method is difficult to translate to the robot following scenario. In our setup, we decide to either let the robot operate on its own or give full control to the user.

\subsection{Tactile Haptic Interfaces}

Traditional kinesthetic haptic feedback devices are capable of displaying a wide range of forces and information through grounded mechanical linkages. However, they are not portable and, therefore, cannot be used in the context of mobile robot-human interaction. Cutaneous, or tactile, haptic 
feedback devices often have smaller size and do not need to be grounded. A review of tactile and wearable haptic interfaces can be found in Ref. [27].

Vibration is a popular type of tactile haptic feedback for various applications, including enhancing stiffness rendering and contact display [44], and providing information about surface texture and roughness [7]. Vibration is also the most commonly used haptic feedback for human-machine interaction. Researchers have explored the idea of using vibrotactile feedback on wrists and other parts of the body as guidance for movement [16, 25, 37]. A single vibrotactile actuator cannot display directional information by itself. Multiple actuators can be combined, but it has been shown that it is difficult to differentiate between vibration actuators that are in close proximity to each other [20].

In this article, we implemented a haptic interface using asymmetric vibrations based on our previous work [8]. It has been shown that asymmetric acceleration pulses can create an ungrounded pulling sensation due to nonlinear perception of acceleration [1,2]. Various mechanisms have been designed to generate asymmetric acceleration, including a mass-spring system [38], a handle that is moved asymmetrically [42], and linear vibration actuators driven with asymmetrically-timed current pulses [3, 28]. Similar to Ref. [3], our haptic interface uses voice coil actuators driven by current pulses, because it is compact, portable, and creates a very noticeable sensation.

\section{ROBOT FOLLOWER}

In this section, we describe the robot platform and the human-following algorithm. Various human detection and following methods have been previously developed, as introduced in Section 2. Some of the methods are simple in which the robot heads toward the human all the time, while some are sophisticated and involve probabilistic inference and motion planning. Here, we implement a simple human-following algorithm that does not actively avoid obstacles. We chose this implementation for a number of reasons. First, the goal of our work is to facilitate human-robot interaction with communication rather than to improve human-following algorithms. Second, more complex algorithms such as avoiding obstacles with motion planning introduce great uncertainties on when the robot would fail. A simpler algorithm enables us to design experiments that are repeatable. Finally, it is not unreasonable to assume simple human-following behavior because it matches the level of performance of some existing commercial products.

We use a Turtlebot 2 as the mobile robot platform. Turtlebot 2 is a low-cost, personal robot kit with open-source software. The robot is equipped with a Microsoft Kinect sensor. We use an ASUS Zenbook UX303UB as the onboard computer to process RGB-D data from the Kinect and to control the robot. All the algorithms described in this and the next section were implemented in Ubuntu 14.04 LTS with the Robot Operating System (ROS Indigo).

\subsection{Following and Tracking Algorithms}

We implemented a simple human-following control algorithm that aims to keep the robot at a desired distance from the human and always facing toward the human:

$$
\begin{aligned}
v & =k_{p, v}\left(\hat{y}-y_{d}\right)+k_{d, v} \dot{\hat{y}} \\
\omega & =k_{p, \omega} \hat{x}+k_{d, \omega} \dot{\hat{x}},
\end{aligned}
$$

where $v, \omega$ are the commanded linear and angular velocities, respectively; $(\hat{x}, \hat{y})$ is the position of the human in the robot's reference frame; $k_{p, v}, k_{d, v}, k_{p, \omega}$, and $k_{d, \omega}$ are controller gains ( $p$ is proportional and $d$ is derivative); and $(\dot{\hat{x}}, \dot{\hat{y}})$ is the velocity of the human with respect to the robot. We use numerical differentiation to calculate the velocity and smooth it using a low pass filter. 
(a)

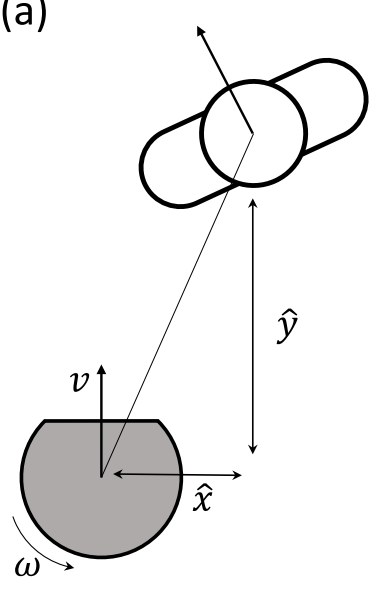

(b)

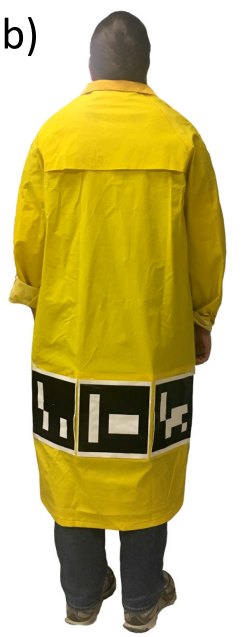

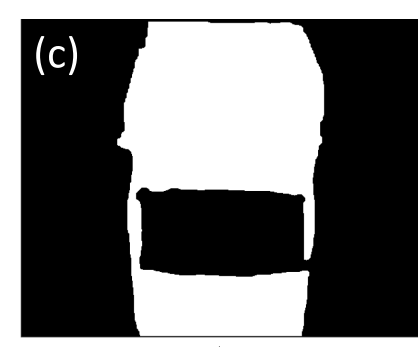

(d)

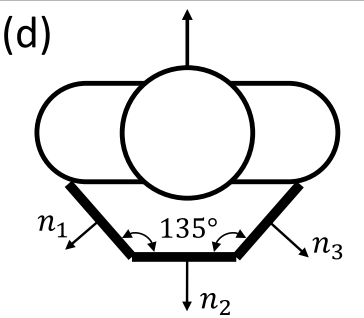

Fig. 2. (a) Geometric relationship between the robot and the human. $\hat{x}, \hat{y}$, and $\theta$ denote the relative position and orientation of the human in the robot's reference frame. $v$ and $\omega$ denote the linear and angular velocity of the robot. (b) Yellow jacket and 2D markers for human tracking. (c) Image mask obtained after applying color thresholding. (d) Diagram showing the positions of the markers. The markers on each side are oriented $\pm 135^{\circ}$ from the one in the middle.

To obtain the relative position of the human, we developed a tracking algorithm based on color segmentation and 2D marker detection. The algorithm is outlined here:

(1) Convert the RGB image to HSV color space, and threshold with HSV range [20, 100, 100] $[30,255,255]$

(2) Remove noise with morphological filters, find the largest contour, and calculate its centroid

(3) Obtain the average distance $\hat{y}$ of the largest contour using the depth image

(4) Convert the pixel positions to physical positions:

$$
\hat{x}=\frac{\hat{y} x_{p}}{f_{w}}
$$

where $x_{p}$ is the position of the centroid in the image, and $f_{w}$ is the focal length of the camera.

(5) Calculate the orientation of the human from the pose of the detected 2D markers

In the last step, the orientation is obtained by taking the weighted average of the poses of the detected markers. We used three ArUco markers [13] rigidly attached to each other, as shown in Figure 2(a)-(c). Each marker is oriented $135^{\circ}$ from its neighbor. The weights of the markers are calculated as:

$$
w_{i}= \begin{cases}2.0 & \text { if }\left|\angle \mathbf{n}_{i}\right|<45^{\circ} \\ 1.0 & \text { if }\left|\angle \mathbf{n}_{i}\right| \geq 45^{\circ}\end{cases}
$$

where $\mathbf{n}_{i}$ is the surface norm of the marker $i, \angle \mathbf{n}_{i}$ is the angle of the surface norm, and $\angle \mathbf{n}_{i}=0$ when the marker is parallel to the camera. To calculate the weighted average of orientations, we implemented the optimization method [17]:

$$
\bar{R}=\arg \min _{R} \sum_{i=1}^{n} w_{i} d_{L}\left(R, R_{i}\right)^{2}
$$




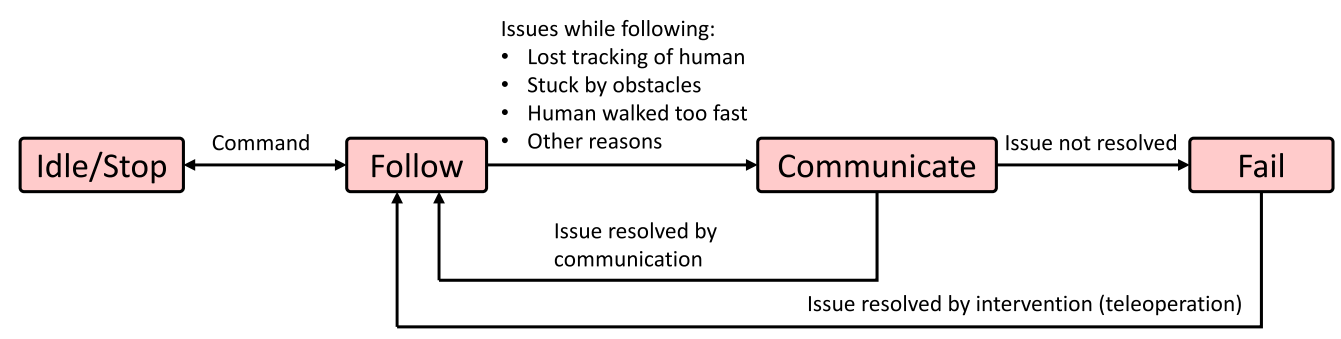

Fig. 3. State machine that defines the robot follower's behavior. High-level description of transition conditions are placed next to the arrows that connect the states. In the "Fail" state, the human user can intervene by teleoperating the robot.

where $\bar{R}$ is the Karcher mean of the rotations, $R_{i}$ is the orientation of marker $i$, and $d_{L}\left(R, R_{i}\right)$ represents the geodesic distance between the two orientations $R$ and $R_{i}$. The orientation $\bar{R}$ is used later to compute the appropriate direction for haptic feedback.

We implemented a simple state machine to enable the robot follower to communicate with the user. Figure 3 shows the structure of the state machine. During normal operation, the user can command the robot to start and stop following. When the robot follower encounters an issue that it can't resolve on its own, it communicates with the user by sending a haptic signal. Some issues may be resolved by communication (for example, in the case of human walking too fast, if the user slows down after receiving a haptic warning signal, the issue will be resolved), and the robot then resumes following. If the issue is not resolved, the robot stops and enters the "Fail" state. In the Fail state, the robot can be teleoperated by the user, and will resume following once the issue is resolved.

The simple following algorithm will not work in a few scenarios, causing the robot to enter the Fail states. Firstly, the algorithm cannot handle losing tracking of the human leader. If the tracking is reported lost for a period longer than a threshold value $t_{t h}$, the robot enters the Fail state. Because the algorithm does not consider obstacle avoidance, the robot will enter the Fail state when it hits an obstacle. This is detected with the bumpers on the robot base. We also consider the robot as being stuck when the robot cannot track the commanded velocity. The robot may also fail to follow if the user walks too fast (faster than the robot's physical speed limit). There are other reasons that the robot may fail, and we will discuss more about failure modes in Section 5.

\section{THE COMMUNICATION DEVICE}

This section describes the design and working principles of the holdable mechatronic device used for communication. As shown in Figure 1, the device consists of two main components: the haptic interface and the command interface. The haptic interface is used for displaying messages sent by the robot when it enters unwanted states. The command interface can be used to control and teleoperate the robot follower when necessary.

\subsection{Haptic Interface}

As described in Section 2, we use asymmetric vibrations to create an ungrounded pulling sensation in different directions. The vibration is created by voicecoil actuators driven by the step-ramp current pulses shown in Figure 4(c). As determined in previous work [8], in order to create an asymmetric acceleration, the actuator should quickly move in the desired direction of the pulling sensation in a short period of time (step phase), and then slowly return in the opposite direction (ramp phase). The step and ramp durations were empirically chosen to be $t_{1}=4 \mathrm{~ms}$ and $t_{2}=15 \mathrm{~ms}$ to produce the most noticeable pulling sensation. 
(a)

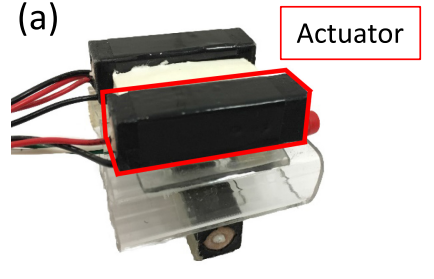

(c)

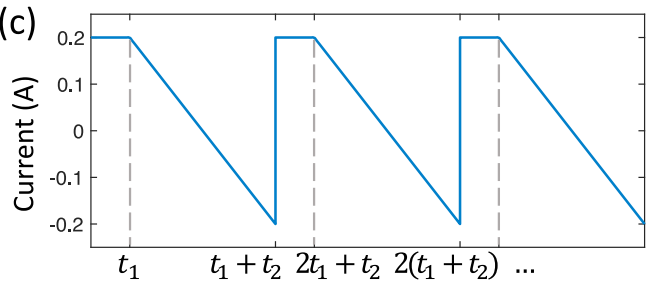

(b)

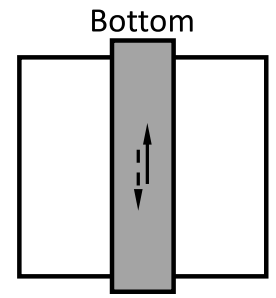

(d)

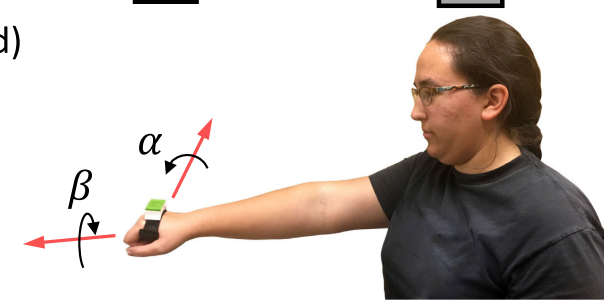

Fig. 4. (a) The haptic interface hardware. The device is held in a pinch grasp, where the thumb contacts the actuator on the bottom. (b) Bottom-up and top-down view of the haptic device, showing one actuator on the bottom and two actuators on the top. Arrows show the directional cues that each actuator can display. Turning directions can be rendered by combining opposite cues from two actuators. (c) The waveform of the control current that is used to generate asymmetric vibrations. (d) The command interface hardware. The device is strapped onto user's hand and can sense the tilting angles of the hand.

Each actuator is capable of displaying two opposite directions by inverting the current signal. To render more than two directions, multiple actuators need to be carefully placed to minimize interference, while maximizing the stimulation to fingertips. Figure 4(a) and (b) show the haptic interface. Three actuators are used; one is placed on the bottom of the device, and the other two actuators are mounted on the top, parallel to each other. We use an acrylic tube as the device base because it is lightweight yet rigid enough to transmit vibration.

We use this prototype to display four distinct directions: forward, backward, clockwise, and counter-clockwise. Figure 4(b) illustrates the working principle of the device. The actuator on the bottom can create forward and backward pulling sensations. The two actuators on the top can vibrate in opposite directions, and their combination creates a twisting sensation that is similar to a torque. During operation, the device is held in a pinch grasp, as shown in Figure 4(a). The thumb should be parallel to and in contact with the actuator on the bottom. Each of the other fingers (except for the little finger) should be in contact with both actuators on the top.

\subsection{Command Interface}

The purpose of the command interface is to enable the human user to control or provide information to the robot follower. We implemented a gesture-based teleoperation interface using an on-body 9-axis IMU. As shown in Figure 4(d), the device is strapped to the back of the user's hand and can sense the pose of the hand. We use the roll $(\alpha)$ and pitch $(\beta)$ angles for controlling the robot follower. The commanded linear velocity is calculated as:

$$
v= \begin{cases}0 & |\alpha| \leq \alpha_{\min } \\ k_{\alpha}\left(\alpha-\operatorname{sgn}(\alpha) \alpha_{\min }\right)+v_{\text {offset }} & \alpha_{\min }<|\alpha| \leq \alpha_{\max } \\ v_{\max } & |\alpha|>\alpha_{\max }\end{cases}
$$

This acts like a proportional controller with deadband and saturation. $\alpha_{\min }$ and $\alpha_{\max }$ are threshold values of the pitch angle, which control the width of the deadband and saturation, respectively. $k_{\alpha}$ is a proportional gain that is used to calculate the commanded velocity from the pitch angle. The 


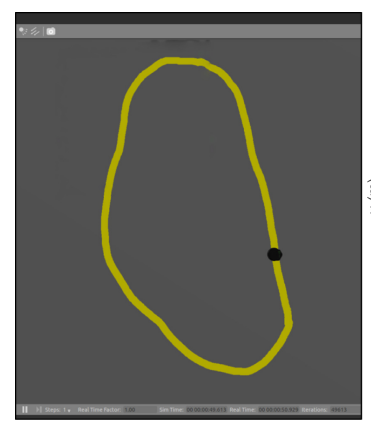

(a)

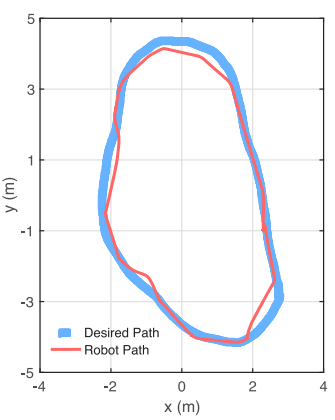

(b)

Fig. 5. (a) Top view of the virtual world with the desired path for the pre-study. The black circle is the robot. During the study, the users were presented with a zoomed-in view centered at the robot. (b) Sample trajectory of the simulated robot. Blue is the desired path, and red is the robot's path.

commanded angular velocity is calculated similarly using the roll angle $\beta$, which is used to control the direction of the robot.

\subsection{Pre-Study of the Haptic and Command Interfaces}

We conducted a pre-study to validate the functionality of the two components of the communication device, and to determine if it is intuitive and easy for users to learn. Five right-handed users participated in the pre-study, after giving informed consent. The protocol was approved by the Stanford University Institutional Review Board. The pre-study consisted of two parts:

4.3.1 Haptic Interface. We tested if users could correctly distinguish the four directions (forward, backward, clockwise, counter-clockwise) rendered by the haptic interface. Before the experiment started, a short training session was given to each user to help them become familiar with the haptic interface. Each user completed 40 trials in total, and the directions were pseudorandomized. In each trial, the vibration was pulsed three times ( 1 second each time), with 1 second pauses in between. The users were then asked to specify the direction displayed. All five users were able to correctly identify all of the directions displayed by the haptic interface $100 \%$ of the time.

4.3.2 Command Interface. In this part, each user teleoperated a simulated Turtlebot 2 to follow a given path in a virtual world using the device. We used Gazebo 2.2 in ROS Indigo for the simulation. Figure 5(a) shows the simulated world, where the desired path is rendered in yellow. Each user was given a two-minute training session to learn how to use the device to control the simulated robot. Then, the user was asked to teleoperate the robot to follow the path for one loop. All the users completed the path-following task successfully within two minutes and reported that the interface was easy to use. Figure 5(b) shows a sample trajectory of the virtual robot teleoperated by a user.

These results indicate that the communication device is straightforward to learn and use.

\section{USER STUDY I: MULTI-TASKING STUDY}

We conducted two sets of user studies to evaluate the effectiveness of the communication scheme and the device. In this section, we describe the first study, in which we focus on scenarios where the users are engaged in multiple tasks and mentally distracted from the robot follower. Our goal was to test if the communication device improves users' awareness of the robot's status and reduces robot following failures. 


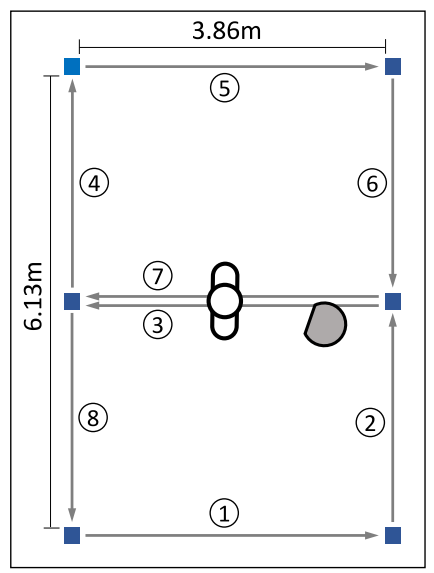

(a)

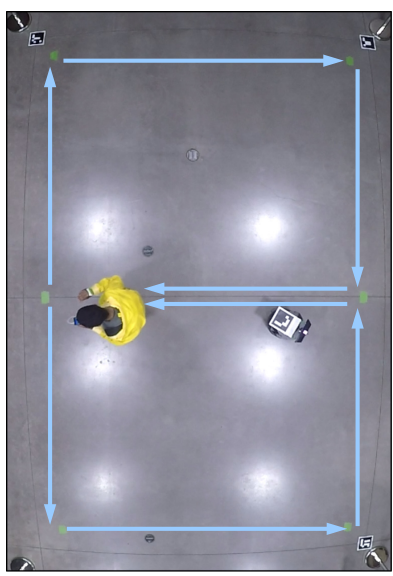

(b)

Fig. 6. Top view of the experimental setup for Study I. (a) Configuration of the experiment field. Blue squares are the waypoints of the desired path. The arrows indicate the direction that the human should follow. (b) Picture of the actual experiment field with the user and the robot follower.

\subsection{Setup}

The experimental setup for this study is illustrated in Figure 6, where the user was asked to follow a path with the robot follower while giving equal weight to the following objectives:

- Follow the given path as closely as possible. The users were instructed to walk through all six waypoints in a "figure 8" pattern, as indicated by the segments (1)-(8)in Figure 6, and repeat the same pattern until instructed to stop (after 5 minutes).

- Complete math problems on a smartphone. This is to simulate the cognitive load of simultaneously performing other tasks. For example, when shopping, people need to search for items, read tags, and remember prices. It has been shown that processes involving the manipulation of symbolic information (digits, words) require the use of working memory [10]. In this study, two-digit addition problems were used.

- Make sure that the robot keeps following. If the robot fails to follow, the user need to use the command interface to help the robot resume following as soon as possible. More details are provided on potential causes of robot failure in the next sub-direction.

During the entire experiment, the user needs to hold the communication device. When the robot fails to follow, the device will generate haptic feedback based on the failure mode. The user can then teleoperate the robot and command the robot to resume following by pressing a button on the device.

\subsection{Experimental Conditions}

We created two scenarios where the robot may fail to follow the user. These scenarios are designed to resemble failures that can happen in real-world applications:

-Scenario 1: Human walks too fast: Most mobile robots, including the Turtlebot 2 used here, have a physical speed limit that is lower than human walking speed. In this study, the robot follower stops following when the user maintains a walking speed faster than a threshold for 1 second. Before the robot enters the failure state, a haptic signal is displayed to the user to warn him or her. The signal is a backward tug with the asymmetric vibration 
Table 1. Summary of Conditions for User Study I

\begin{tabular}{|c|c|c|}
\hline Session No. & Communication & Scenario/Failure Mode \\
\hline 1 & Off & Human too fast \\
\hline 2 & On & Human too fast \\
\hline 3 & Off & Random \\
\hline 4 & On & Random \\
\hline
\end{tabular}

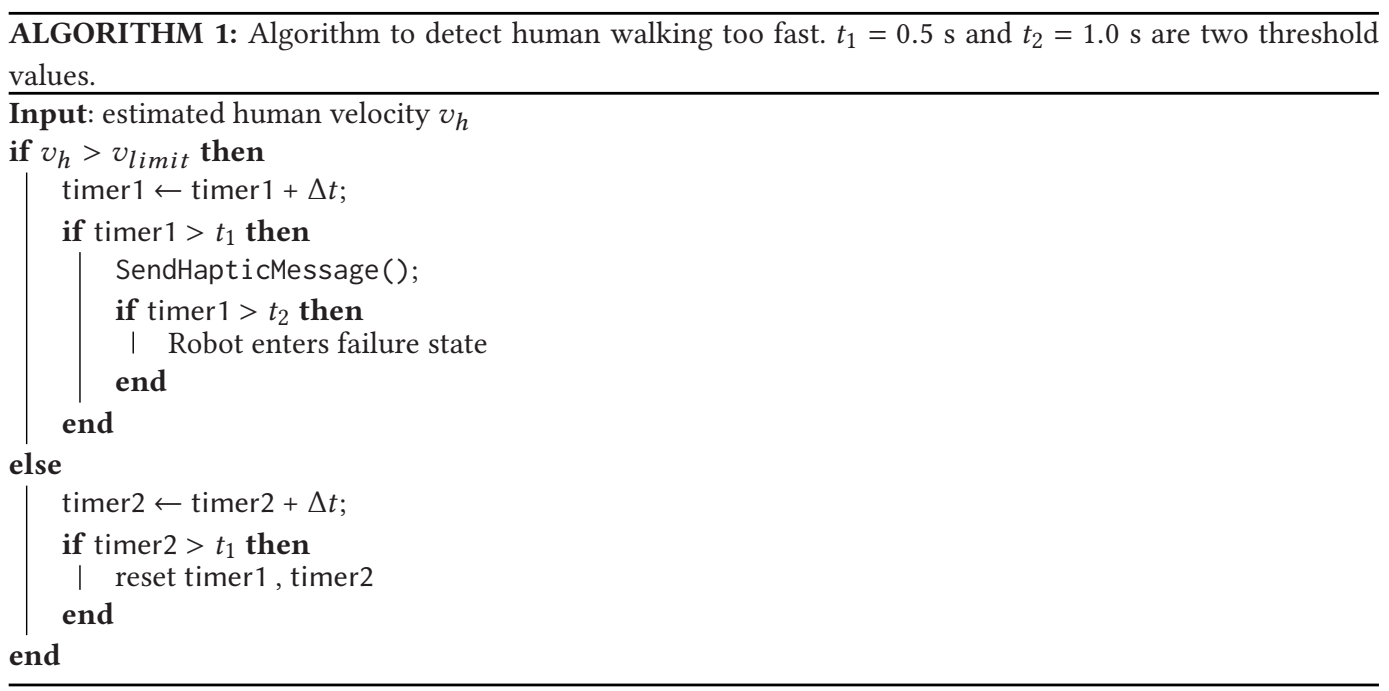

interface and is triggered when the user maintains a fast speed for 0.5 seconds. The exact implementation is described by Algorithm 1.

-Scenario 2: Randomly: We manually set the robot to fail after a random period of time between 20-40 seconds. The purpose of this failure mode is to simulate unpredictable failures that may happen in the real world, such as hitting an undetectable obstacle on the ground, or temporary sensor failure due to external interference. These failures are hard to foresee or prevent.

For each scenario, we ran one session with communication turned on, and one without communication. In the case where the communication is turned off, the user still holds the device, but no feedback is provided, and gesture teleoperation is disabled. A total number of four sessions were tested for each participant, and they are summarized in Table 1.

In both scenarios when the communication is turned on, a directional haptic signal will be displayed to the user when the robot enters the failure state. The haptic signal creates either a clockwise or a counter-clockwise torque sensation, depending on the relative position of the robot with respect to the human. To calculate the relative position, we performed a coordinate transformation from the robot's frame of reference to the user's frame of reference:

$$
\begin{aligned}
& x_{r}=-\hat{x} \cos (\theta)-\hat{y} \sin (\theta) \\
& y_{r}=\hat{x} \sin (\theta)-\hat{y} \cos (\theta)
\end{aligned}
$$

If $x_{r}>0$, a clockwise cue would be displayed, and if $x_{r}<0$, a counter-clockwise cue would be displayed. Compared to the backward signal that warns the user of walking too fast, this signal is stronger in magnitude and is repeated twice for each failure event. 


\subsection{Procedure}

At the start of the experiment, each user was given a short training session on the use of the haptic interface and the command interface, and a 90 -second warm-up with the math problems. The user was then allowed to walk around with the robot follower for a short period, to become familiar with the robot and the task field. Each user completed four sessions with different scenarios and communication conditions as shown in Table 1. For each session, the user performed the previously described task for 5 minutes.

In the sessions where the communication was off, the user had to rely solely on visual information to tell if the robot was in a failure state. An LED placed on the top of the robot could indicate the robot's status by different colors: green represented normal state and purple represented a failure.

When the communication was turned on, the holdable device alerted the user of robot failure events as described in Section 5.2. The user could use the communication device to teleoperate the robot after it failed. For example, the robot would not resume following if it was too far from the user. In this scenario, the user could manually command the robot to move closer and then resume following.

After finishing the experiment, each user filled out a survey with three questions:

(1) Rate the usefulness of having haptic feedback from 1 to 5 (1 meaning that it made performance worse; 5 meaning that it improved performance).

(2) Rate the usefulness of being able to teleoperate the robot from 1 to 5 .

(3) How often could you interpret the directional cue from haptic feedback? Choices are $0 \%$, $25 \%, 50 \%, 75 \%$, and $100 \%$ of the time.

The entire experiment was recorded by an overhead camera for post-processing. We used a GoPro Hero 4 Black to record the video at $30 \mathrm{~Hz}$. The robot status was recorded at $50 \mathrm{~Hz}$.

Eight users (4 male, 4 female), ages from 24 to 33, all right-handed, participated in the experiment after giving informed consent. The protocol was approved by the Stanford University Institutional Review Board. All users completed Scenario 1 (Sessions 1 and 2) first and then Scenario 2 (Sessions 3 and 4). The order of providing the communication is pseudo-randomized: for each scenario, half of the users had the communication on first, while the other half had the communication off first.

\subsection{Analysis and Results}

5.4.1 Trajectories and Robot Status. Figure 7(a) shows average trajectories of a single user under Session 4. The gray line represents the user's average trajectory and the red line represents the robot's average trajectory. To obtain the average trajectory, we first processed the video and extracted the position of the user and the robot at each frame. The user's location was calculated by tracking the color of the jacket on the user, and the robot's position was calculated by detecting the 2D marker attached as described in Section 3. Then, the position data is filtered with a thirdorder Butterworth filter with cutoff frequency at $5 \mathrm{~Hz}$. Finally, we applied k-means clustering using Euclidean distance to group the positions into 100 clusters, and we represent each cluster with its centroid. These centroids formed the average trajectory. Figure 7(a) also shows the correspondence between the user's and the robot's positions with the dashed line segments. Each segment connects a pair of centroids from the user's and the robot's trajectories.

Figure 7(b) shows the robot's status over time for all four sessions from a single user. Comparing Sessions 1 and 2, it can be observed that with haptic feedback, the robot failed fewer times. Comparing Sessions 3 and 4, we observe that the response time to the robot failure event is much shorter when the haptic feedback is enabled (Communication On). These observations can be confirmed by the statistical tests on the data of all the users. 

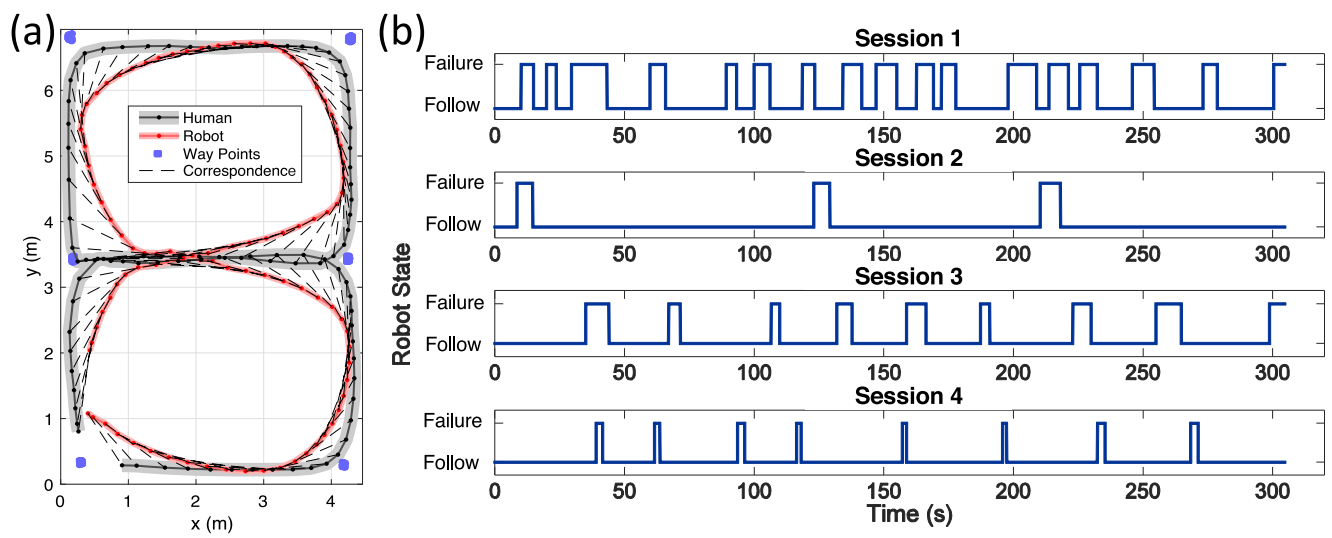

Fig. 7. Sample trajectory and robot states in Study I. (a) Sample trajectory from a user in Session 4. The gray line represents the user's trajectory, and the red line represents the robot's trajectory. Blue squares are the desired waypoints. The dashed line segments show the correspondence of the user and the robot's position at the same time for the sampled locations. (b) Robot status versus time for the four different sessions from a user. See Table 1 for details of each session.

5.4.2 Performance Metrics. Three quantitative metrics were used to evaluate the performance:

- Number of failure states: The number of times the robot failed to follow. This metric is only relevant for Sessions 1 and 2. On average, the robot failed the same number of times in Sessions 3 and 4 because the failures were random. To calculate this metric, we counted the number of times the robot status transitioned from "follow" to "failure" state. We did not count time where the robot failed at the end of the trial.

- Duration of failure states: The duration of time the robot remained in the failure state before the user revived it. For each user, we calculated the average duration of failure states as the total duration divided by the number of failure states.

- Normalized math performance: Here, we are only interested in comparing the relative performance across sessions, since the users' abilities and strategies for doing the math problems varied greatly. The normalized math performance was calculated as:

$$
\frac{\text { Number of problems solved in Session } k}{\text { Total number of problems solved/4 }}
$$

where the denominator is the average number of problems solved in one session.

We used a paired t-test to test for statistically significant differences in performance metrics between the Communication On and Off conditions. We consider the two scenarios (failure modes) as independent sub-studies, and only compare the Communication On and the Communication Off condition for each failure mode. Figure 8(a) and (b) show the average number and duration of failure states in the different experimental conditions. When haptic feedback was enabled, the robot failed significantly fewer times, compared to the Communication Off condition $(t(7)=2.5184, p=0.0399)$. For failure mode 2 (robot failed randomly), the average duration of failure states when haptic feedback was provided (Communication On) was significantly shorter than when haptic feedback was not provided (Communication Off) $(t(7)=4.0458, p=0.0049)$. However, there is no statistically significant difference between the sessions in Scenario 1 (robot fails when the human walks too fast). Figure 8(c) shows the average normalized math performance for 


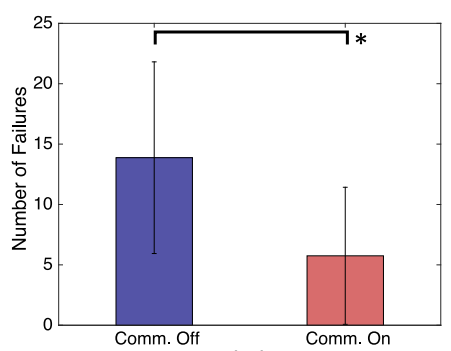

(a)

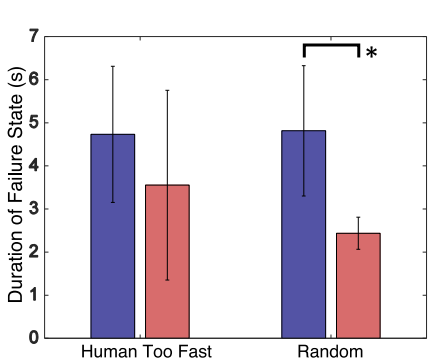

(b)

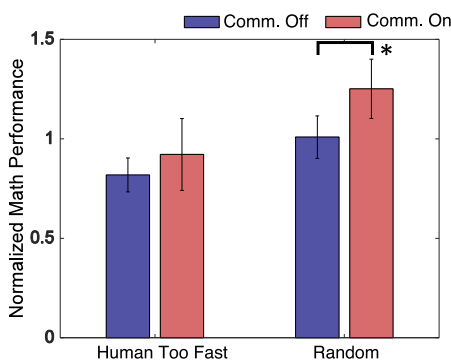

(c)

Fig. 8. Statistics of Study I. (a) Average number of times that the robot failed in Session 1 (Communication On) and Session 2 (Communication Off). (b) Average time spent by the user to help the robot recover from a failure state for all sessions. Blue represents Communication On, and red represents Communication Off. (c) Normalized math performance for all sessions. Brackets indicate statistical significance at 0.05 level $\left({ }^{*}\right)$. The error bars represent the standard deviation.

(a)

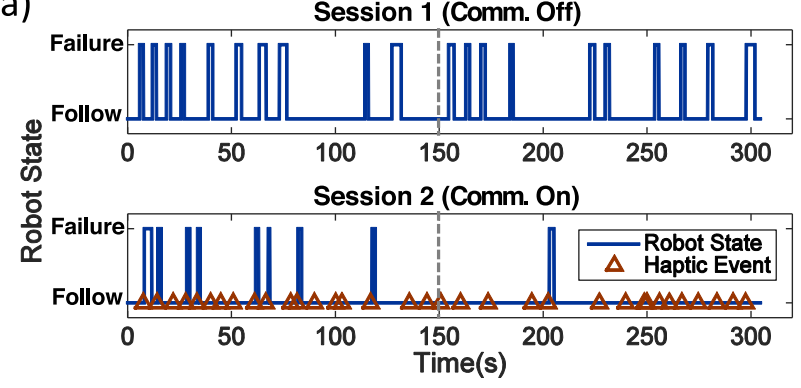

(b)

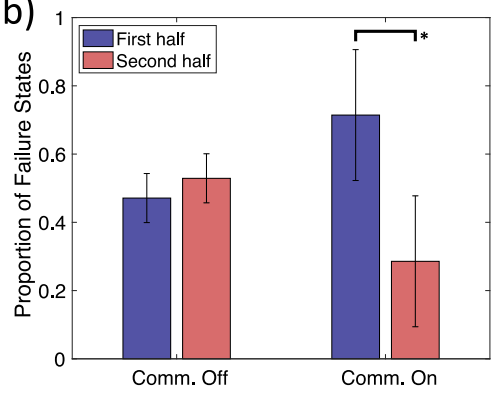

Fig. 9. Comparison of the first and the second half of each trial in Study I. (a) Sample robot states vs. time and haptic feedback event vs. time for Session 1 and Session 2 for a single user. (b) Normalized number of times that robot failed in the first half of the trial vs. the second half of the trial. The bracket indicates statistical significance at 0.05 level $\left({ }^{*}\right)$. The error bars represent the standard deviation.

all sessions. Similar to Figure $8(\mathrm{~b})$, there is a statistically significant difference between Session 3 and 4 (failure mode 2) $(t(7)=-4.0152, p=0.0051)$, but not Session 1 and 2 (failure mode 1).

5.4.3 Learning Effects. We investigated the learning effect during a session by comparing the number of failure states in the first half and the second half of the trial. Figure 9(a) shows sample robot states versus time in Sessions 1 and 2 for a single user. The haptic feedback event is also marked in Session 2 when haptic feedback was enabled. Here, we only show haptic cues that alerted the user that they were walking too fast. The first half and the second half of each trial is divided by $t=150 \mathrm{~s}$, as indicated by the dashed line. Comparing the first and second half of Session 1, we observe that the number of failure states is approximately the same. In Session 2, on the other hand, the robot failed far fewer times in the second half. We performed statistical tests on the average number of failure states in the first and second half of each trial. Similar to the math performance, we normalize the number of failure states because the users' individual responses varied greatly. The normalized number of failure states, or proportion of failure states in the first half, is calculated as:

$$
\bar{N}_{1, k}=\frac{\text { number of failure states in first half of Session } k}{\text { number of failure states in Session } k}
$$



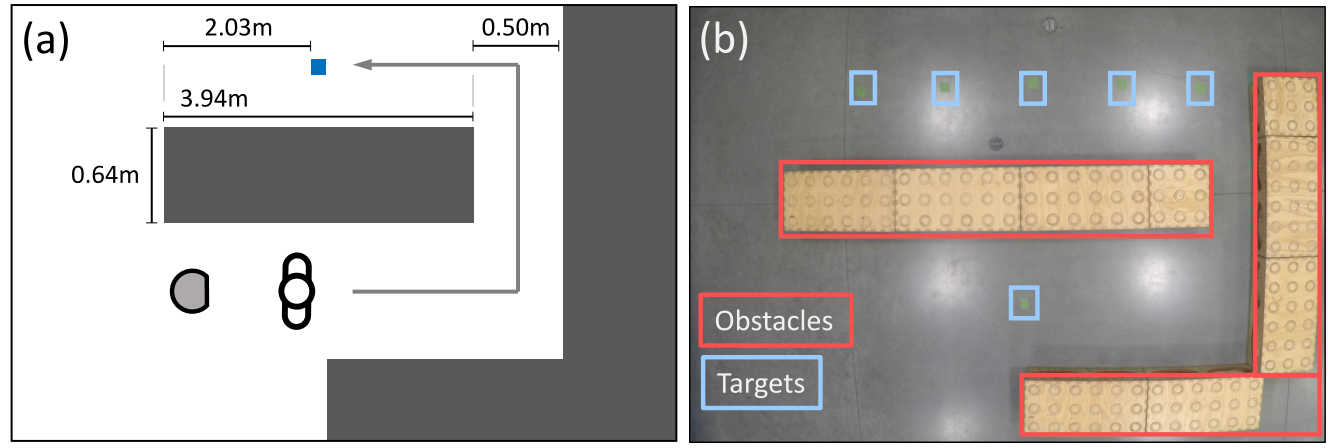

Fig. 10. Experimental setup of Study II. (a) Setup for the first part of Study II. The blue square marks the goal position, and arrows indicate the valid path. (b) Picture of the actual experiment field, obstacles, and the targets are marked by red and blue boxes, respectively.

The proportion in the second half is simply $\bar{N}_{2, k}=1-\bar{N}_{1, k}$. Another interpretation of $\bar{N}_{1, k}$ is the estimated probability of the robot failing in the first half in Session $k$.

Figure 9(b) summarizes the results. When communication is on, the normalized number of failure states in the second half is statistically less than the first half $(t(7)=3.4136, p=0.0112)$. When communication is off, there is no statistically significant difference between the first and the second half.

5.4.4 Survey Results. Average ratings for the usefulness of haptic feedback and teleoperation were 4.63 and 3.25, respectively. Contradicting our expectation from the pre-study, users were able to interpret the directional cue only about $44 \%$ of the time on average.

\section{USER STUDY II: NAVIGATION STUDY}

In Study I, we showed that haptic feedback was very effective in improving users' awareness of the robot's status, especially when the users were under cognitive load. However, because of the simple setup, the study did not address the scenarios where the users need to teleoperate the robot. In the second study, we investigated how people would use the command interface to teleoperate and help the robot to avoid obstacles.

\subsection{Experimental Setup and Procedure}

In this study, the user was asked to walk from a starting point to a destination point in the setup environment shown in Figure 10. The user had to avoid the obstacles indicated by the gray areas in the figure. Since our human-following controller did not incorporate active collision avoidance, the robot would likely get stuck and fail to follow the user if it hit an obstacle. In this case, the user would receive a clockwise or counter-clockwise haptic cue similar to Study I. The user could then use the command interface to teleoperate the robot follower to help it get around the obstacles. The robot could also be manually switched to teleoperation mode.

There were two parts in this study:

- Part 1: As shown in Figure 10(a), in each trial, the user started from the initial position and walked to the goal position along a path indicated by the arrows. The desired path went through a narrow passage, which is very challenging for the robot. The user's task is to get to the goal position with the robot follower. There are two conditions for this part: 


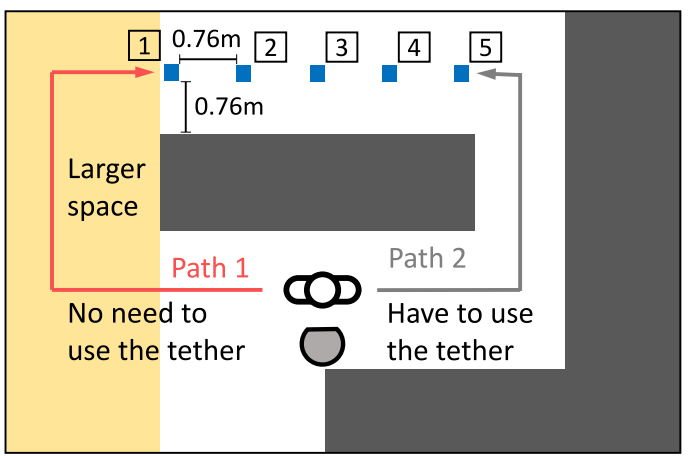

Fig. 11. Setup for the second part of Study II. Blue squares are the goal positions and are numbered 1 to 5 from left to right. Red arrows and gray arrows represent two choices of paths.

- Communication Off: The user does not receive haptic feedback and has no direct control of the robot follower. In order to avoid getting the robot stuck by the obstacles, the user has to carefully choose their path. If the user is not able to reach the goal position in 30 seconds with the robot follower, the trial is considered a failure (it is extremely unlikely that the user can succeed in a trial if they get the robot stuck once, which usually happens within 30 seconds). Through pilot testing, we found that the user's trajectories were consistent while repeating the condition (no learning effect observed), and the user would get frustrated after a few trials. For this reason, we decide to limit this condition to three trials.

- Communication On: The user receives haptic feedback and can use the command interface to teleoperate the robot follower. In this condition, the user can always reach the target with the robot, as they have direct control over the robot. We are interested to study how fast the user can learn and adapt to the communication interface. Based on pilot testing, we set 10 trials for this condition.

- Part 2: Figure 11 shows the setup for the second part of the study. In each trial, the user is given a target position numbered from 1 to 5 . The user can choose between Path 1 on the left (red arrows) and Path 2 on the right (gray arrows). If Path 1 is chosen, the user is not allowed to use the command interface. The space on the left side is wide enough for the robot to avoid collision. If Path 2 is chosen, the user can use the command interface to teleoperate the robot to get through the narrow passage. The goal of each trial was to reach the target position as fast as possible. Each user completed five trials, one for each target position. The order of the five targets were pseudo-randomized.

In each trial of Parts 1 and 2, the robot began in the idle/stop state. The user switches the robot into following state by pressing the button attached to the haptic interface twice. The user pressed the button twice again upon reaching the target position, to indicate that the trial is finished. When ending the trial, the user needs to make sure that the robot is following.

After the experiment, each user was asked to fill out a survey, with two questions that were identical to the first two questions in the survey from Study I.

Eight users (3 male, 5 female), ages from 23 to 33, all right-handed, participated in the study after giving informed consent. Similar to Study I, the experiments were recorded by an overhead camera. All of the users completed Part 1 of the study first, and half of them were given the Communication Off condition before the Communication On condition. 

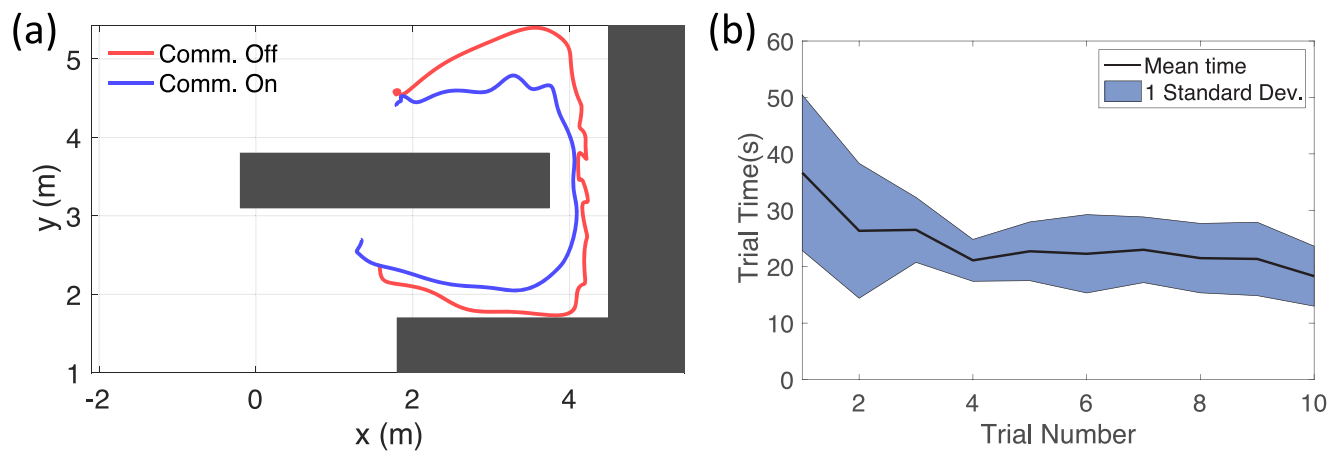

Fig. 12. (a) Sample trajectories from one user in Part 1. Blue trajectory is from Communication On condition, and red trajectory is from Communication Off condition. (b) Average time spent by the users to get to the target position vs. trial number in the "Communication On" condition in Part 1 of Study II. The blue region represents one standard deviation around the mean trial time.

\subsection{Analysis and Results}

6.2.1 Benefit of Teleoperation. In the Communication Off condition, the robot became stuck on the obstacles more than $60 \%$ of the time (15 out of 24 trials). In the Communication On condition, users were able to reach the target every time by teleoperating the robot to move through the narrow passage. Figure 12(a) presents sample trajectories from the two conditions. We can see that when communication is off, the user had to take unnatural paths to try to guide the robot away from the corners.

6.2.2 Learning Effects. We studied how fast the users learned to use the command interface by looking at the average completion time of a trial versus trial numbers, as shown in Figure 12. The completion time of a single trial is calculated as $t_{\text {end }}-t_{\text {start }}$, where $t_{\text {start }}$ and $t_{\text {end }}$ are the time when the user pressed the button to start and stop the robot, respectively. The completion time decreases relatively fast initially, then plateaus after about four trials.

6.2.3 User Preference. Figure 13(a) shows the trajectories of two users who chose different paths of the same target. The red and gray trajectories correspond to Path 1 and Path 2 shown in Figure 11. For target 3, Path 1 is longer than Path 2, because the user decided to go farther from the obstacle to avoid the robot becoming stuck.

We compare the number of users who chose Path 1 to the number of users who chose Path 2, for targets 1 to 5 . The result is shown in Figure 13(b). For targets 1-3, most users chose Path 1, while for target 5 , the majority of users chose Path 2. For target 4, half of the users chose Path 1 , and the other half chose Path 2. We also computed the path length difference between Path 1 and Path 2. For each target position, the path length difference is calculated as the average path length of users who chose Path 1 minus the average path length of users who chose Path 2. Note that for target 1 , none of the users chose Path 2, so we extrapolated the value from the path lengths of the other 4 targets.

6.2.4 Survey Results. The average user ratings of the usefulness of haptic feedback and teleoperation were 3.38 and 5.00 , respectively.

\section{DISCUSSION}

In this section, we discuss the effectiveness of the proposed communication scheme, focusing on how it affected task performance and changed the way that users interacted with the robot follower. 

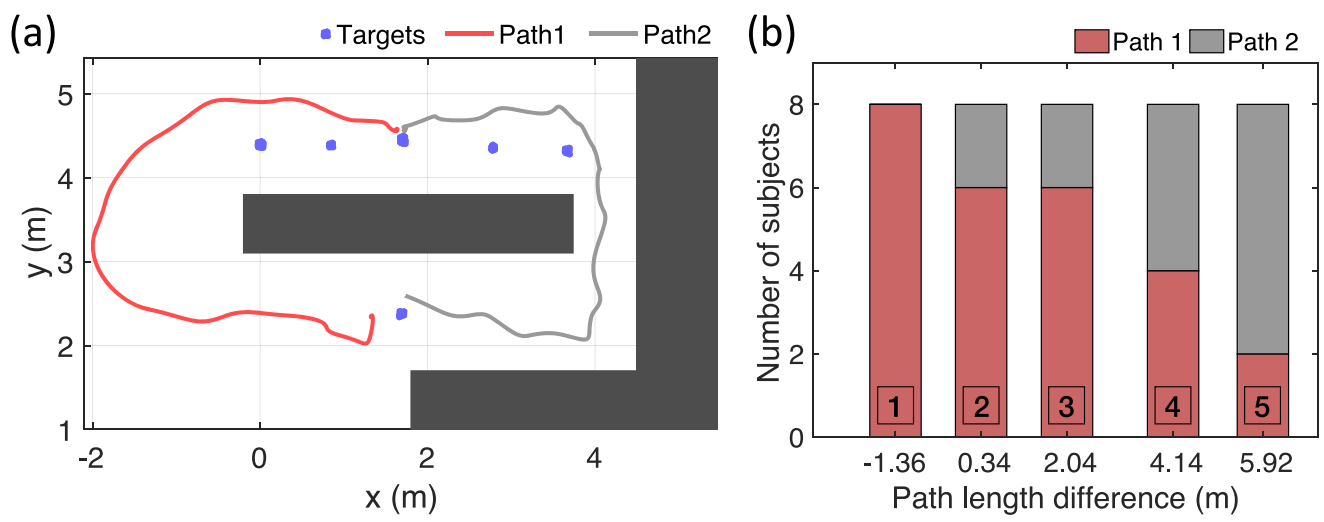

Fig. 13. (a) Sample trajectories from two users who chose different paths to the same target (Target 3) in Study II. The two paths are represented by the red and gray trajectories. The starting point and the targets are indicated by the blue squares. Note that the robot trajectories are not shown here. (b) Number of users who chose Path 1/Path 2 vs. path length difference (Path 1 minus Path 2) in Study II. The red part represents users who chose Path 1, while the gray part represents users who chose Path 2.

\subsection{Study I: Multi-Tasking}

We showed that with haptic feedback alerts, the users were able to better control their walking speed, resulting in significantly fewer failure states for the robot follower, compared to without haptic feedback. Similar results have been reported in Ref. [36], where the authors demonstrated that users could regulate their speed and turning radius to reduce the formation error by using a haptic bracelet. These results indicate that haptic feedback is a promising method for conveying information about constraints or rules of interaction with mobile robotic systems.

More interestingly, we found that with haptic feedback, users not only violated the speed limit fewer times, but also learned and adapted to this constraint by reacting to the haptic cues. From Figure 9 we see that when communication was enabled, the robot failed to follow for significantly fewer times in the second half of the trials. This is not observed in the condition where communication was off. This difference was observed because in this study, the users were engaged in multiple tasks: path following and solving math problems. With high mental load, the users tended to maintain a constant walking speed, regardless of the robot's maximum speed, which is reflected by the evenly distributed robot failure events in Session 1 (Comm. Off). However, when provided with haptic feedback as a warning for walking too fast, the users were able to develop reactional strategies and reduce their walking speed upon receiving the haptic cues. In Figure 9(a), we observe that although the robot follower failed for fewer times in the second half of Session 2, the number of haptic events in the first and second half of the trial were similar. The users were taking advantage of the haptic information to better control their speed.

Haptic feedback is also effective in improving the user's awareness of the robot's status while lowering the user's mental effort. In the scenarios where the robot follower failed randomly (Sessions 3 and 4), providing haptic feedback significantly reduced the users' response time, as shown in Figure 8(b). While the random failure scenario may seem unrealistic, the purpose is to simulate unpredictable failures in real-world applications. In these applications, the users' visual and audio input channels may already be over-stimulated, and thus, may not be able to notice the robot follower's failure. The haptic channel, on the other hand, can provide intuitive feedback about the robot's status. Users also completed significantly more math problems when the communication 
was on, as shown in Figure 8(c). This result indicates that using haptic feedback can help offload the effort of paying attention to the robot follower, so that users can perform better on other tasks.

Through the post-experiment survey, we found that all the users thought having haptic feedback improved their overall performance (three users responded " 4 " and five users responded " 5 " to the first survey question). However, survey results also showed that, on average, the users can successfully interpret the left and right directional cues only about $44 \%$ of the time. In contrast, our pre-study results showed that users can recognize the directional cues $100 \%$ of the time when not performing other tasks. There are a few possible reasons for this contradiction. First, the pre-study was conducted while the user was sitting still. In the actual experiment, the user was constantly walking, which introduced movements and accelerations to the hand, in addition to the asymmetric vibration. The additional movements may confuse the user and affect their ability to distinguish the directional cue. In addition, the directions rendered by the haptic interface are relative to the hand pose. In the pre-study, users held the hand in a fixed pose. However, in the real experiment, users did not keep a fixed hand pose while walking. Finally, the user had to perform multiple tasks in the experiment. High mental load may also create difficulties for recognizing the directional cues.

We also found that the ability to teleoperate the robot is not critical in this study, and users only rated 3.25 for the usefulness of teleoperation (seven out of eight users selected " 3 "). Although the users were able to teleoperate the robot with the command interface in Session 2, only one user actually used it. When the robot failed to follow and was too far from the user, it would not resume following until the user got close enough. In this case, the user either needed to walk back to the robot or to command the robot to come closer. We found that seven out of the eight users chose to walk to the robot. In this particular study, it was easier for the user to move than to teleoperate the robot. There may be applications where it is more helpful to command the robot to move closer. In the second study, we showed a scenario where the command interface was proven to be useful.

\subsection{Study II: Navigation}

The haptic interface provides a one-way communication channel from the robot to the user, while the command interface provides the channel for the opposite direction-from the user to the robot. It enables the user to send information to the robot or to directly take control of the robot. This can be useful when the robot's autonomous behavior is not satisfying or sufficient. A good example is the scenario in Study II where the user had to walk through a narrow passage with the robot follower (shown in Figure 11). Although the human-following algorithm we implemented does not perform obstacle avoidance, other algorithms may fail in this scenario as well due to uncertainties in sensing and actuation.

Without the command interface, the robot became stuck on the obstacles more than $60 \%$ of the time (15 out of 24 trials). When using the command interface, the user and the robot follower could successfully reach the goal position in every trial. Before the study, only one training session was given to each user, in which they used the command interface to teleoperate the robot in free space for 1 minute. During the study, we found that all users were able to teleoperate the robot to move through the narrow passage using the command interface given the short learning period. Figure 12(b) showed that the average trial completion time dropped quickly initially, and then plateaued after about four trials. The result indicates that users were able to learn to use the command interface efficiently within a few trials.

To further clarify whether this decrease in completion time is due to users' adaptation to the command interface or due to improvement in their trajectories, we analyzed their trajectories and whether they teleoperated the robot in each trial in the Communication On condition. We found that users' trajectories remained consistent throughout the trials (similar to the blue trajectory 
in Figure 12(a)). In almost every trial (78 out of 80 ), users relied on teleoperation to guide the robot through the narrow passage. Therefore, we conclude that the learning effect observed in Figure 12(b) is indeed due to users' adaptation to the command interface. The gesture teleoperation was enabled in two different cases (when communication was on): when the robot entered a failure state, or when the user manually switched the robot into teleoperation mode by pressing a button on the command interface. In the first case, the user would receive a haptic feedback that alerted them of the failure state before they could start teleoperation. Interestingly, we observe that some users relied on the haptic feedback to decide if the robot was in trouble and needed to be teleoperated, while other users decided to manually switch the robot to teleoperation mode whenever they approached the narrow passage. The reason behind this is that the robot had a high probability of failure upon entering the passage. So the risk-averting users chose to teleoperate the robot to avoid hitting the obstacles, while the risk-accepting users chose to give the robot a try and only intervene when necessary. As a result, in the post-experiment survey, haptic feedback was rated to be not very helpful in this study (3.38 on average). In scenarios where robot failures are hard to predict, we expect most users to rely on haptic feedback instead of being risk-averting and teleoperating the robot frequently.

Sometimes using the command interface may not be the only choice. In the second part of Study II, the users were given two paths to the target positions: one that requires the use of the command interface and one that does not. Depending on the target position, either path could be the shorter one. Here, we are interested in investigating how users chose between the two options given different target positions. It can be observed from Figure 13(b) that the shorter Path 2 is compared to Path 1, the more likely that users will choose Path 2 and use the command interface. When Path 2 is much shorter than Path 1 (5.92m difference as in the case of Target 5), most users preferred Path 2 . When Path 2 is only slightly shorter ( 2m, Target 3$)$, most users chose Path 1 so that the robot would have little challenge following them. These results demonstrate that the users in general would prefer to use the command interface when it improves their path efficiency by a certain threshold, even though teleoperating the robot requires additional time and effort. In our specific setup, this threshold is around $4 \mathrm{~m}$ (Figure 13(b)). In a real-world application, the differences among alternative paths are usually larger than this experiment, so the command interface could be very helpful in this scenario.

\subsection{Limitations and Future Work}

The current design of the communication system has several limitations revealed by the studies, motivating future work.

First, the current haptic interface requires the user to hold it in order to provide effective feedback. This limits the user's ability to perform manipulation tasks with both hands. In addition, holding the device in hand for a long period may cause fatigue. We are exploring different haptic feedback technologies to make wearable haptic interfaces that can generate similar cues. An example of such device is presented in Ref. [9].

Some users also reported that the command interface is difficult to use, primarily due to the fact that the controls were offset/rotated depending on the relative orientation of the robot. Ideally, the user only needs to send high-level instructions instead of performing full teleroperation to help the robot. In future work, we are interested in developing command strategies that are intuitive and require minimum effort. In conjunction, we will work on algorithms that enable the robot to understand these commands and act accordingly.

Finally, the experimental setups are simple compared to real-world scenarios. The reason we start with these experiments is to obtain repeatable results and measure performance in a controlled environment. Testing in the real world introduces additional, partially confounding 
factors. Nevertheless, experiments in more realistic settings should be carried out to evaluate the real-world usefulness of the bi-directional communication device.

\section{CONCLUSION}

In this article, we presented a new communication scheme for mobile robot-human interaction. We described the design of a holdable mechatronic device that enables bi-directional communication between human and a robot follower. The device consists of two parts: the haptic interface, which can pass information from the robot to the human user in the form of haptic feedback; and the command interface, which can be used by the user to teleoperate the robot. We conducted experiments to evaluate the effectiveness of the communication scheme and the holdable device.

Our user studies showed that the proposed method can facilitate interaction between the user and the robot follower and can improve their performance in various tasks. We showed that haptic feedback increases users' awareness of the robot follower's status and lowers their mental effort, thus, helping the users perform better on other tasks. It also allows the users to react quickly to unexpected events such as robot failure. The command interface (gesture teleoperation) can be useful when the robot's autonomous behavior is not satisfying or sufficient. We demonstrated that users can successfully use the command interface to teleoperate the robot follower when it fails in challenging environments. Combining the haptic and command interface, the bi-directional communication creates a tight bond between the human user and the robot follower.

\section{REFERENCES}

[1] Tomohiro Amemiya, Hideyuki Ando, and Taro Maeda. 2005. Phantom-DRAWN: Direction guidance using rapid and asymmetric acceleration weighted by nonlinearity of perception. In International Conference on Augmented TeleExistence. 201-208.

[2] Tomohiro Amemiya, Hideyuki Ando, and Taro Maeda. 2005. Virtual force display: Direction guidance using asymmetric acceleration via periodic translational motion. In IEEE World Haptics Conference. 619-622.

[3] Tomohiro Amemiya and Hiroaki Gomi. 2014. Distinct pseudo-attraction force sensation by a thumb-sized vibrator that oscillates asymmetrically. In International Conference on Human Haptic Sensing and Touch Enabled Computer Applications. 88-95.

[4] Edwin Babaians, Navid Khazaee Korghond, Alireza Ahmadi, Mojtaba Karimi, and Saeed Shiry Ghidary. 2015. Skeleton and visual tracking fusion for human following task of service robots. In RSI International Conference on Robotics and Mechatronics. 761-766.

[5] Kim Baraka, Stephanie Rosenthal, and Manuela Veloso. 2016. Enhancing human understanding of a mobile robot's state and actions using expressive lights. In IEEE International Symposium on Robot and Human Interactive Communication. IEEE, 652-657.

[6] Luca Consolini, Fabio Morbidi, Domenico Prattichizzo, and Mario Tosques. 2008. Leader-follower formation control of nonholonomic mobile robots with input constraints. Automatica 44, 5 (2008), 1343-1349.

[7] Heather Culbertson, Juliette Unwin, and Katherine J. Kuchenbecker. 2014. Modeling and rendering realistic textures from unconstrained tool-surface interactions. IEEE Transactions on Haptics 7, 3 (2014), 381-393.

[8] Heather Culbertson, Julie M. Walker, and Allison M. Okamura. 2016. Modeling and design of asymmetric vibrations to induce ungrounded pulling sensation through asymmetric skin displacement. In IEEE Haptics Symposium. 27-33.

[9] Heather Culbertson, Julie M. Walker, Michael Raitor, and Allison M. Okamura. 2017. WAVES: A wearable asymmetric vibration excitation system for presenting three-dimensional translation and rotation cues. In Proceedings of the 2017 CHI Conference on Human Factors in Computing Systems. ACM, 4972-4982.

[10] Meredyth Daneman and Philip M. Merikle. 1996. Working memory and language comprehension: A meta-analysis. Psychonomic Bulletin \& Review 3, 4 (1996), 422-433.

[11] Aveek K. Das, Rafael Fierro, R. Vijay Kumar, James P. Ostrowski, John Spletzer, and Camillo J. Taylor. 2002. A visionbased formation control framework. IEEE Transactions on Robotics and Automation 18, 5 (2002), 813-825.

[12] D. B. Edwards, T. A. Bean, D. L. Odell, and M. J. Anderson. 2004. A leader-follower algorithm for multiple AUV formations. In IEEE/OES Autonomous Underwater Vehicles. 40-46.

[13] S. Garrido-Jurado, R. Mu noz Salinas, F. J. Madrid-Cuevas, and M. J. Marín-Jiménez. 2014. Automatic generation and detection of highly reliable fiducial markers under occlusion. Pattern Recognition 47, 6 (2014), 2280-2292. 
[14] Rachel Gockley, Jodi Forlizzi, and Reid Simmons. 2007. Natural person-following behavior for social robots. In ACM/IEEE International Conference on Human-Robot Interaction. 17-24.

[15] H.-M. Gross, Ch Schroeter, Steffen Mueller, Michael Volkhardt, Erik Einhorn, Andreas Bley, Ch Martin, Tim Langner, and Matthias Merten. 2011. Progress in developing a socially assistive mobile home robot companion for the elderly with mild cognitive impairment. In IEEE/RSF International Conference on Intelligent Robots and Systems. IEEE, 24302437.

[16] Wenjiang Guo, Wei Ni, I-Ming Chen, Zhong Qiang Ding, and Song Huat Yeo. 2009. Intuitive vibro-tactile feedback for human body movement guidance. In IEEE International Conference on Robotics and Biomimetics. 135-140.

[17] Richard Hartley, Jochen Trumpf, Yuchao Dai, and Hongdong Li. 2013. Rotation averaging. International fournal of Computer Vision 103, 3 (2013), 267-305.

[18] Nobutaka Hirai and Hiroshi Mizoguchi. 2003. Visual tracking of human back and shoulder for person following robot. In IEEE/ASME International Conference on Advanced Intelligent Mechatronics. 527-532.

[19] Fumiaki Hoshino and Kazuyuki Morioka. 2011. Human following robot based on control of particle distribution with integrated range sensors. In IEEE/SICE International Symposium on System Integration. 212-217.

[20] Lynette A. Jones and Nadine B. Sarter. 2008. Tactile displays: Guidance for their design and application. Human Factors 50, 1 (2008), 90-111.

[21] Marin Kobilarov, Gaurav Sukhatme, Jeff Hyams, and Parag Batavia. 2006. People tracking and following with mobile robot using an omnidirectional camera and a laser. In IEEE International Conference on Robotics and Automation. $557-562$.

[22] T. John Koo and Shahram M. Shahruz. 2001. Formation of a group of unmanned aerial vehicles (UAVs). In American Control Conference. 69-74.

[23] Carlo Masone, Antonio Franchi, Heinrich H. Bülthoff, and Paolo Robuffo Giordano. 2012. Interactive planning of persistent trajectories for human-assisted navigation of mobile robots. In IEEE/RSf International Conference on Intelligent Robots and Systems. 2641-2648.

[24] C. Masone, P. R. Giordano, H. H. Bülthoff, and A. Franchi. 2014. Semi-autonomous trajectory generation for mobile robots with integral haptic shared control. In IEEE International Conference on Robotics and Automation. 6468-6475.

[25] Michael Matscheko, Alois Ferscha, Andreas Riener, and Manuel Lehner. 2010. Tactor placement in wrist worn wearables. In International Symposium on Wearable Computers. 1-8.

[26] Kazuyuki Morioka, Joo-Ho Lee, and Hideki Hashimoto. 2004. Human-following mobile robot in a distributed intelligent sensor network. IEEE Transactions on Industrial Electronics 51, 1 (2004), 229-237.

[27] Claudio Pacchierotti, Stephen Sinclair, Massimiliano Solazzi, Antonio Frisoli, Vincent Hayward, and Domenico Prattichizzo. 2017. Wearable haptic systems for the fingertip and the hand: Taxonomy, review, and perspectives. IEEE Transactions on Haptics 10, 4 (2017), 580-600.

[28] Jun Rekimoto. 2013. Traxion: A tactile interaction device with virtual force sensation. In Annual ACM Symposium on User Interface Software and Technology. 427-432.

[29] Stephanie Rosenthal, Joydeep Biswas, and Manuela Veloso. 2010. An effective personal mobile robot agent through symbiotic human-robot interaction. In International Conference on Autonomous Agents and Multiagent Systems. 915922.

[30] Stephanie Rosenthal and Manuela Veloso. 2010. Using symbiotic relationships with humans to help robots overcome limitations. In Workshop for Collaborative Human/AI Control for Interactive Experiences.

[31] Stephanie Rosenthal, Manuela M. Veloso, and Anind K. Dey. 2011. Task behavior and interaction planning for a mobile service robot that occasionally requires help. In AAAI Conference on Automated Action Planning for Autonomous Mobile Robots. AAAI Press, 14-19.

[32] Nicholas Roy, Gregory Baltus, Dieter Fox, Francine Gemperle, Jennifer Goetz, Tad Hirsch, Dimitris Margaritis, Michael Montemerlo, Joelle Pineau, Jamie Schulte, et al. 2000. Towards personal service robots for the elderly. In Workshop on Interactive Robots and Entertainment, Vol. 25. 184.

[33] Junji Satake and Jun Miura. 2009. Robust stereo-based person detection and tracking for a person following robot. In ICRA Workshop on People Detection and Tracking. 1-10.

[34] Stefano Scheggi, Marco Aggravi, and Domenico Prattichizzo. 2017. Cooperative navigation for mixed human-robot teams using haptic feedback. IEEE Transactions on Human-Machine Systems 47, 4 (2017), 462-473.

[35] Stefano Scheggi, Francesco Chinello, and Domenico Prattichizzo. 2012. Vibrotactile haptic feedback for human-robot interaction in leader-follower tasks. In International Conference on Pervasive Technologies Related to Assistive Environments. 51:1-51:4

[36] Stefano Scheggi, Fabio Morbidi, and Domenico Prattichizzo. 2014. Human-robot formation control via visual and vibrotactile haptic feedback. IEEE Transactions on Haptics 7, 4 (2014), 499-511.

[37] Fabrizio Sergi, Dino Accoto, Domenico Campolo, and Eugenio Guglielmelli. 2008. Forearm orientation guidance with a vibrotactile feedback bracelet: On the directionality of tactile motor communication. In IEEE RAS/EMBS International Conference on Biomedical Robotics and Biomechatronics. 433-438. 
[38] Takuya Shima and Kenjiro Takemura. 2012. An ungrounded pulling force feedback device using periodical vibrationimpact. In International Conference on Human Haptic Sensing and Touch Enabled Computer Applications. 481-492.

[39] Dominik Sieber, Selma Music, and Sandra Hirche. 2015. Multi-robot manipulation controlled by a human with haptic feedback. In IEEE/RSf International Conference on Intelligent Robots and Systems. 2440-2446.

[40] Aaron St Clair and Maja Mataric. 2015. How robot verbal feedback can improve team performance in human-robot task collaborations. In ACM/IEEE International Conference on Human-Robot Interaction. ACM, 213-220.

[41] Atsushi Tani, Gen Endo, Edwardo F. Fukushima, Shigeo Hirose, Masatsugu Iribe, and Toshio Takubo. 2011. Study on a practical robotic follower to support home oxygen therapy patients-development and control of a mobile platform. In IEEE/RSF International Conference on Intelligent Robots and Systems. IEEE, 2423-2429.

[42] Hanns W. Tappeiner, Roberta L. Klatzky, Bert Unger, and Ralphs Hollis. 2009. Good vibrations: Asymmetric vibrations for directional haptic cues. In World Haptics Conference. 285-289.

[43] Sebastian Thrun. 2004. Toward a framework for human-robot interaction. Human-Computer Interaction 19, 1-2 (2004), 9-24.

[44] Parris Wellman and Robert D. Howe. 1995. Towards realistic vibrotactile display in virtual environments. ASME Dynamic Systems and Control Division 57, 2 (1995), 713-718.

[45] Takashi Yoshimi, Manabu Nishiyama, Takafumi Sonoura, Hideichi Nakamoto, Seiji Tokura, Hirokazu Sato, Fumio Ozaki, Nobuto Matsuhira, and Hiroshi Mizoguchi. 2006. Development of a person following robot with vision based target detection. In IEEE/RSF International Conference on Intelligent Robots and Systems. 5286-5291.

[46] Woo-han Yun, Dohyung Kim, and Jaeyeon Lee. 2010. Person following with obstacle avoidance based on multi-layered mean shift and force field method. In IEEE International Conference on Systems Man and Cybernetics. 3813-3816.

Received January 2018; revised July 2018; accepted July 2018 